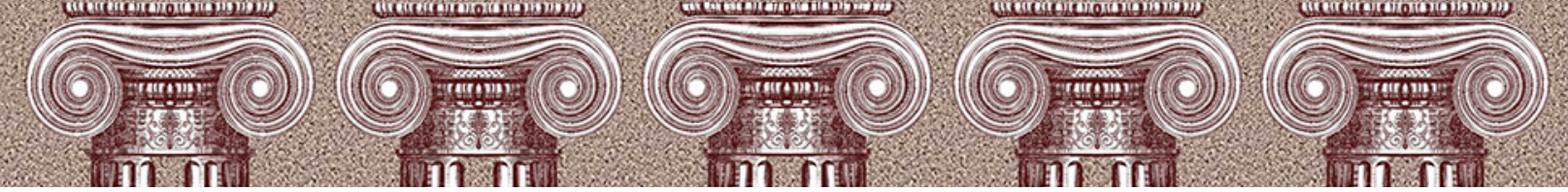

Artículo de investigación E13A05: Proyecto "Análisis del desarrollo del proceso emprendedor en el Sector Turístico de Esquel” - Universidad Nacional de la Patagonia San Juan Bosco. * Recibido: 14.08.2018.

Aceptado versión final: 25.01.2019. * JEL: L26; B59; D21; L83. * Pp. 159-181. * doi:10.33571/teuken.v10n15a7

\title{
Caracterización de emprendedores y emprendimientos del sector turístico de Esquel, Argentina
}

\section{Characterization of entrepreneurs and entrepreneurships of the tourism sector of Esquel, Argentina}

\author{
María Victoria Fernández - María Celeste Ramírez - Silvia Mabel López \\ Mariana Gauna Lavayén - Rubén Ángel César
}

\section{ARGENTINA}

Resumen: El artículo tiene por objetivo caracterizar los emprendedores y emprendimientos del sector turístico de Esquel, Argentina, desde el enfoque del emprendedorismo. Se parte de la hipótesis de que la mayoría de emprendimientos son Pymes familiares con bajo potencial de crecimiento. Para el desarrollo metodológico se aplicó una encuesta semiestructurada a una muestra de emprendedores del sector turístico, representativa del total y se caracterizaron como style life, que son aquellos que buscan alcanzar su desarrollo personal antes que desarrollar emprendimientos dinámicos, porque su fin último es sostener un estilo de vida determinado. La mayoría de las empresas son familiares, de tamaño pequeño y mediano, que trabajan en pequeña escala para sostenerse en el mercado.

Palabras clave: Emprendedorismo; cultura emprendedora; emprendedor turístico; motivaciones.

Abstract: The objective of the paper is to characterize the entrepreneurs and entrepreneurs of the tourism sector in Esquel, Argentina, from an entrepreneurial perspective. It is based on the hypothesis that the majority of enterprises are family SMEs with low growth potential. For the methodological development a semistructured survey was applied to a sample of entrepreneurs of the tourism sector, representative of the total and characterized as style life, which are those that seek to achieve their personal development rather than develop dynamic ventures, because their ultimate goal is to sustain a determined lifestyle. Most companies are family, small and medium-sized, that work on a small scale to sustain themselves in the market.

Keywords: Entrepreneurism; entrepreneurial culture; tourist entrepreneur; motivations.

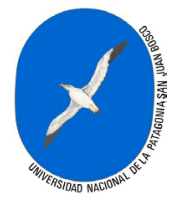

María Victoria Fernández es Licenciada en Economía y Especialista en Docencia Universitaria. Actualmente, es Profesora Adjunta de la Facultad de Ciencias Económicas y Jefe de Trabajos Prácticos de la Facultad de Ingeniería, de la Universidad Nacional de la Patagonia San Juan Bosco, Delegación Esquel.

Contacto: mvfernan@hotmail.com 


\section{Caracterização dos empreendedores e empreendedores do sector turístico de Esquel, Argentina}

Resumo: O objetivo do artigo é caracterizar os empreendedores e empresários do setor de turismo em Esquel, Argentina, a partir de uma perspectiva empreendedora. Baseia-se na hipótese de que a maioria das empresas são PMEs familiares com baixo potencial de crescimento. Para o desenvolvimento metodológico foi aplicado um survey semiestruturado a uma amostra de empreendedores do setor turístico, representativos do total e caracterizados como estilo de vida, que são aqueles que buscam atingir seu desenvolvimento pessoal ao invés de desenvolver empreendimentos dinâmicos, pois seu objetivo final é sustentar estilo de vida determinado. A maioria das empresas é familiar, pequena e média, que trabalha em pequena escala para se sustentar no mercado.

Palavras-chave: Empreendedorismo; cultura empreendedora; empreendedor turístico; motivações.

\section{Ubicación en el contexto}

$\mathrm{E}$

n la región cordillerana de la provincia de Chubut, ubicada en la Patagonia argentina, una de las actividades que modelan la economía regional es el turismo. La presencia de la cordillera de los Andes, con sus exuberantes bosques templados y la conformación de valles, con ríos y lagos, hacen del paisaje un excepcional atractivo que anualmente es visitado por más de 100000 amantes de la naturaleza. Las principales actividades que se desarrollan son la pesca, el trekking y senderismo, la visita al Parque Nacional Los Alerces, principalmente, durante la temporada estival, y el esquí, durante el invierno. El desarrollo de estas actividades es motorizado, mayormente, por prestadores radicados en la localidad, tanto del ámbito privado como público, cuya tarea e interacción dan forma a la experiencia que viven los turistas.

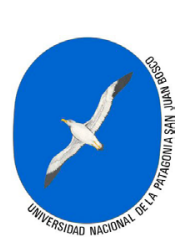

\footnotetext{
María Celeste Ramírez es Licenciada en Administración. Actualmente, es Profesora y Auxiliar Docente de 1ra. Categoría de Planeamiento y Evaluación de Proyectos, de la Universidad Nacional de la Patagonia San Juan Bosco, Delegación Esquel.

Contacto: ramirezceleste@yahoo.com.ar

Silvia Mabel López es Profesora Adjunta de Planeamiento y Evaluación de Proyectos y Auxiliar Docente de 1ra. Categoría de Estadística, de la Universidad Nacional de la Patagonia San Juan Bosco, Delegación Esquel.

Contacto: silmalopez@hotmail.com
}

Mariana Gauna Lavayén es Licenciada en Sociología. Actualmente, es Profesora de la Facultad de Ciencias Económicas, de la Universidad Nacional de la Patagonia San Juan Bosco, Delegación Esquel.

Contacto: gauna4196@yahoo.com.ar

Rubén Ángel Cesar es Licenciado en Economía Industrial. Actualmente, es Investigador docente del Programa Desarrollo Emprendedor (PRODEM UNGS), del Instituto de Industria de la Universidad Nacional de General Sarmiento.

Contacto: rcesar@ungs.edu.ar 
Desde 1947, la región Patagónica recibe diversas corrientes migratorias que se asientan en las ciudades más pobladas de las provincias que la componen, provenientes en los primeros años de regiones rurales y urbanas, pero a partir de 1970, de los centros urbanos más poblados, dándose un fenómeno a nivel nacional en el que crece la presión migratoria hacia ciudades de tamaño intermedio y se reduce en la región metropolitana (Torrado, 1992: 89). Durante los años 50 se produjo un proceso migratorio importante para la ciudad producto de la política de estado denominada Desarrollismo, cuya idea central era que el país debía promover un proyecto integral que expandiera la economía más allá de los clásicos elementos primarios, con referencia a la producción de materias primas (Secretaría de Cultura y Educación, 2009: 164). Si bien el turismo en Esquel y su zona de influencia comenzó a manifestarse de manera creciente y sostenida a mediados de los años 60, su definición como eje del turismo en la zona se fortaleció con la creación de la Dirección Provincial de Turismo en el año 1964, para ese entonces el sector contaba con actividades turísticas vinculadas al Parque Nacional Los Alerces, creado en la década del 30 que brindaron la oportunidad para su consolidación (CCPITOCH, 2006: 129).

Dada su conformación histórica, el sector turístico de Esquel se caracteriza por la fuerte presencia del sector público, que se constituye como administrador de los principales atractivos naturales, como, por ejemplo, el Parque Nacional, otras Reservas Provinciales y el Centro de Actividades de Montaña La Hoya. El sector privado es quien, mediante la instalación de emprendimientos de mediana y pequeña escala, vinculados al territorio, recibe todos los años a los visitantes, brindándoles los servicios de alojamiento, visitas guiadas, restauración, etc.

\section{Objetivo e hipótesis del trabajo}

En un entorno en el que la demanda turística es acotada y estacional, la hipótesis de investigación apunta a demostrar que el sector empresarial del ámbito turístico está constituido por pequeñas empresas que no aspiran al rápido crecimiento y acumulación de capital, sino que basan su actividad en la especialización y son gerenciadas por emprendedores que priorizan su desarrollo personal y el sostenimiento económico de sus familias, antes que el rápido crecimiento de sus negocios. En esta dirección, el objetivo del artículo es determinar las principales características que describen a los emprendedores y empresas del sector turístico de la localidad de Esquel, desde el enfoque del emprendedorismo para compararlas con la hipótesis planteada.

Este artículo es un primer acercamiento que se inscribe en un proyecto de investigación más ambicioso denominado Análisis del desarrollo 
del proceso emprendedor en el sector turístico de Esquel, ejecutado por la Facultad de Ciencias Económicas de la Universidad Nacional de la Patagonia San Juan Bosco. El objetivo general de este proyecto es describir y analizar particularidades del proceso de desarrollo emprendedor de las empresas del sector turístico de la localidad de Esquel, poniendo énfasis en determinar las principales características de los empresarios y en describir limitantes y aportes del entorno que influyen en las actividades relacionadas con el negocio, siendo el último de estos no abordado en el presente trabajo.

\section{Caracterización de emprendedores y emprendimientos desde el emprendedorismo}

El marco conceptual en el que se encuadra este trabajo es el propuesto por los teóricos del emprendedorismo. Este campo del conocimiento, desde el siglo XVIII y XIX, se ha fundamentado en los aportes de la economía, administración y psicología. En los últimos 30 años, ha alcanzado un fuerte impulso debido a que la creación de nuevas empresas contribuye al crecimiento económico, la generación de puestos de trabajo e innovaciones, el surgimiento de nuevos sectores de actividades de pequeñas y medianas empresas, la regeneración de los tejidos productivos regionales y la canalización de las energías creativas de la sociedad (Kantis y Federico, 2007: 13; Ateljevic \& Li, 2009: 22; Veciana, 1999: 11). Otros autores reconocen que el desarrollo del turismo puede observarse desde la perspectiva de las pequeñas empresas innovadoras, cuya participación en la economía ha contribuido, significativamente, al desarrollo de las economías regionales (Ateljevic, 2009: 149; Solvoll, Alsos \& Bulanova, 2015: 121).

Por su parte, Lebendiker (2013: 14) y Ateljevic \& Li (2009: 22) señalan que muchas teorías han tratado de definir la palabra emprendedor. Algunas de ellas ponen el foco en la persona y sus cualidades, otras se centran más en el entorno y las condiciones que permiten que determinadas sociedades sean más prolíficas para el nacimiento de nuevas empresas. Estas teorías apuntan, por un lado, a presentar una definición, características y cualidades del emprendedor $y$, por el otro, a encontrar una explicación del proceso de creación de empresas y la dinámica del entorno que genera situaciones propicias o desfavorables para la creación, desarrollo y subsistencia de los emprendimientos.

Si bien los científicos han realizado numerosas contribuciones al desarrollo de esta teoría, aún no hay consenso en la definición de lo que es un emprendedor (Ateljevic \& Li, 2009: 22; Solvoll, Alsos \& Bulanova, 2015: 122). Por su parte, Gartner (1988: 57) presenta la relación de tres 
enfoques que pretenden definirlo: aquel que rescata sus cualidades, el que describe sus características y el que analiza su comportamiento. Este autor argumenta que un emprendedor puede tener ciertas cualidades, pero quien tenga esas cualidades o formas de comportarse puede no ser necesariamente un emprendedor. La línea que analiza su comportamiento rescata lo que el emprendedor hace. Entonces, el emprendedor es, en definitiva, quien realiza ciertas acciones que culminan en la creación de un emprendimiento.

Un emprendedor es quien toma la iniciativa, obtiene y organiza los recursos con el fin de lograr un objetivo, administra la organización que creó, posee autonomía en la toma de decisiones y administración de los recursos, y es quien asume el riesgo del negocio (Shapero,1984: 24). De otro lado, Gartner (1988: 59) rescata sus características, señalando que el emprendedor es un individuo que dirige un negocio con el fin de obtener un beneficio y crecer; es innovador y estratega a la hora de administrar el negocio.

Shapero (1984: 28) sostiene que un emprendedor no nace, sino que se hace. Reconoce motivaciones que existen entre quienes son emprendedores, como, por ejemplo, el deseo de ser independientes, un fuerte sentido del control interno y de sus vidas, la tolerancia frente a la ambigüedad. Se trata de personas que pueden trabajar a destajo para llevar adelante su proyecto y poseen altos niveles de energía. Ateljevic \& Li (2009: 23) asumen que toda acción humana es el resultado de factores motivacionales y cognitivos. Shane, Locke \& Collins, (2003: 258) agregan que existen factores externos que juegan un papel determinante al momento de emprender.

Atendiendo a esta diferencia y en reconocimiento de la falta de consenso en torno al concepto de emprendedor, se utilizó como base para desarrollar el perfil del emprendedor turístico en la comunidad de Esquel un enfoque en el que confluyen las características y los comportamientos del emprendedor.

Lebendiker (2013: 17) propone clasificar a los emprendedores, por un lado, como emprendedores por oportunidad, que son quienes emprenden porque han detectado un área de vacancia en el mercado que creen poder cubrir y capitalizar, y, por otro, están los emprendedores por necesidad, que son quienes emprenden frente a una situación de crisis, porque no encuentran otra alternativa para su subsistencia personal o familiar. El autor agrega que la mayoría de los emprendedores por oportunidad suelen ser individuos con cierta formación educativa (secundario, terciario o universitario completo o incompleto), y con algún tipo de red 
de contactos inicial proveniente de sus estudios, trabajo o familia, que los alientan y ayudan en las primeras etapas del proyecto.

Para el caso de emprendimientos turísticos, Parra y Calero (2006: 545) señalan que hay factores que influyen en la elección de este sector y menciona los siguientes: la posibilidad de residir en una zona que se adecue a sus preferencias personales (rural, por ejemplo), el deseo de permanecer o retornar a una propiedad familiar, el perpetuar el legado de su familia, la posibilidad de poner en práctica sus aficiones personales o, simplemente, la posibilidad de desarrollar una actividad que ha podido constituir la ilusión de toda una vida.

De acuerdo con Parra y Calero (2006), se descarta la posibilidad de que en turismo se pueda hablar de emprendedores por necesidad que apuestan al autoempleo, debido a la ausencia de mejores alternativas en el ámbito del empleo en relación de dependencia, y tampoco es frecuente encontrarse con un perfil de emprendedor orientado al crecimiento, regido, principalmente, por la maximización del beneficio, porque, en la mayoría de los casos, prevalecen los objetivos de autonomía y estilo de vida, lo cual solo suele ser compatible con un tamaño reducido de la actividad empresarial. Ateljevic \& Doorne (2000: 381) señalan que los emprendedores crean estas empresas por la necesidad de constituir el estilo de vida elegido, que será aquel en el que las necesidades de la familia, el ingreso y el estilo de vida se equilibren. Un tema clave que rodea estos negocios está relacionado con la supervivencia económica y la viabilidad.

Muchas de estas miradas centran su atención en el concepto de motivación. Robbins y Judge (2009: 176) explican que la motivación es un proceso que involucra la intensidad, dirección y persistencia de un individuo para la consecución de un objetivo. Por su parte, Chiavenatto (2001: 69) explica que un motivo es aquello que impulsa a una persona a actuar de determinada manera. El proceso motivacional comienza con impulsos, que pueden provenir de factores internos (características de la personalidad, capacidad de aprendizaje, percepción), de factores externos (valoración social del emprendedor, condiciones económicas de oportunidad) o de ambos tipos de factores. Estos factores provocan una situación de inconformidad en el estado emocional del individuo, que, a su vez, genera una necesidad. De acuerdo al nivel de tensión que provoque esta situación se produce un comportamiento que conduce a satisfacerla.

Diversas teorías se enfocan en explicar este proceso. Entre las que se puede destacar la Teoría de la Jerarquía de las Necesidades propuesta 
por Maslow. Esta teoría explica que las necesidades humanas están distribuidas en una pirámide, dependiendo de la importancia e influencia que tengan en el comportamiento humano. En la base de la pirámide se encuentran las necesidades más elementales y recurrentes, denominadas necesidades primarias; en la cima se hallan las más sofisticadas y abstractas, llamadas secundarias (Chiavenatto, 2001: 72). En las primeras se encuentran las fisiológicas y de seguridad, y en el segundo grupo las denominadas sociales, de estima y autorrealización. Identificar algunos de los estímulos que dan inicio al ciclo motivacional y que influyen en las personas al momento realizar un emprendimiento permiten caracterizar el origen de este tipo de decisiones que pueden provenir de necesidades de orden inferior (primarias) o superior (secundarias) e identificar a los emprendedores del sector con las categorías que propone Lebendiker (2013: 18).

Desde un enfoque teórico fundado en las ciencias sociales, alternativo al emprendedorismo, Gámez, Ángeles y Juárez (2013) señalan que se le atribuye al capital social la explicación y medio para lograr el desarrollo de ciertas comunidades. Este factor se genera a partir de las relaciones humanas y se define desde los elementos de confianza mutua, reciprocidad, normas efectivas y redes sociales. El capital social se refiere al contexto de sociabilidad que hace que prospere la colaboración y acción colectiva, y que puede permitir que actores individuales aprovechen las oportunidades que surgen en estas relaciones sociales. Desde un enfoque minimalista, que acompaña el enfoque de este trabajo, el capital social se entiende como un recurso intangible, a través del cual las personas acceden a redes personales favorables (Román y Smida, 2009: 17; Ostrom y Ahn, 2003: 160).

La relación entre el empresario creador y la empresa creada determina la existencia de una dinámica y enriquecimiento mutuo entre el capital social del empresario, derivado de sus relaciones sociales previas a la creación de la empresa, y el capital social que la empresa, recientemente creada, debe desarrollar como uno de los recursos raros, escasos e inimitables que sostendrán su ventaja competitiva (Román y Smida, 2009: 17). Las relaciones personales del emprendedor le permiten, o facilitan, la posibilidad de obtener información privilegiada, acceder a conocimiento especializado, reputación y contactos clave que favorecen la puesta en marcha, desarrollo inicial y consolidación del negocio.

En concordancia con los aspectos mencionados con anterioridad, y con el objetivo de caracterizar los emprendimientos, Lebendiker (2013: 18) rescata la existencia de tres tipos: los denominados gacela o de alto impacto, que son empresas que una vez fundadas no paran de crecer y 
que son semilleros de otros emprendimientos; los dinámicos, que son empresas cuyo objetivo es lograr un crecimiento en capital y empleo a una tasa mayor que la del promedio de sus competidores y, finalmente, los emprendimientos de estilo de vida o style life cuyo objetivo no está puesto necesariamente en un ambicioso crecimiento, sino en la búsqueda de un trabajo que permita a su fundador aplicar el conocimiento en un producto o servicio, y, al mismo tiempo, evitar gestionar un número importante de recursos humanos.

En relación con el turismo, Parra y Calero (2006: 544) mencionan que, junto a las importantes dinámicas de integración, expansión internacional y multinacionalización lideradas por las grandes empresas del sector turístico, se observa la emergencia de pequeñas iniciativas empresariales que, habitualmente ligadas a negocios familiares o de carácter autónomo y orientadas a la consecución del autoempleo de sus promotores, logran sostenerse en contextos de globalización. Estas empresas poseen ventajas que favorecen su competitividad, entre ellas, la posibilidad de especialización en segmentos específicos, que conocen en detalle y que pueden atender justamente por su naturaleza de Pymes.

La supervivencia de emprendimientos turísticos se encuentra ligada a la capacidad de sus dueños para propiciar la integración empresarial y la cooperación, la formación de sus recursos humanos y la adaptación de las nuevas tecnologías de la información. La profesionalización de la dirección es la principal estrategia del negocio (Parra y Calero, 2006: 543). Otros autores como Altjevick \& Li (2009: 10) señalan que existen nuevos enfoques que contemplan los valores sociales y culturales como factores de éxito de los emprendimientos turísticos, además del desarrollo y crecimiento de sus negocios.

\section{Metodología}

El objeto de estudio que se abordó son los emprendedores turísticos y empresas de la localidad de Esquel. En concordancia con la Ley Nacional de Turismo $\mathrm{N}^{\circ}$ 25.997, se consideraron emprendedores del sector a aquellos individuos que poseen empresas instaladas en la localidad y que pertenecen a:

- Las empresas directamente vinculadas al turismo: alojamiento, agencias de viaje, transporte (sólo de traslados de turistas y alquiler de vehículos); profesionales de turismo (guías de pesca, licenciados y técnicos, guías en turismo); rental de Ski y escuelas de esquí;

- Las empresas indirectamente vinculadas al turismo: gastronomía con cubiertos y otros servicios tales como negocios de artesanías. 
Se consideró que aquellos que poseen una empresa en el sector son emprendedores, porque han transitado el proceso de identificar, evaluar y aprovechar oportunidades para el desarrollo de nuevos negocios y diseño de productos innovadores y que, a partir de sus competencias personales y de su interacción con el contexto, en un marco de incertidumbre, logran combinar recursos, superar dificultades y establecer vínculos con actores del entorno (César et al. 2016: 4).

De acuerdo con la información obtenida en el organismo de turismo municipal, Esquel cuenta con aproximadamente 190 emprendimientos vinculados en forma directa con el turismo, y cerca de 69 empresas vinculadas en forma indirecta. Algunos emprendedores son dueños de varias empresas dentro y fuera del sector, lo cual dificultó la obtención de un listado de empresarios.

Con el objetivo de caracterizar a los emprendedores y sus emprendimientos, e identificar su relación con el entorno, se diseñó una encuesta semiestructurada de 59 preguntas, siendo las primeras 25 las que apuntan a caracterizarlos. El estudio se realizó entre el 20 de mayo y 10 de junio de 2017. La muestra, seleccionada al azar, fue de 76 emprendedores de la localidad de Esquel. Las actividades de diseño de muestreo se realizaron mediante la aplicación de la técnica probabilística por estratos, con afijación proporcional. Los estratos se conformaron según los grandes rubros mencionados en la Ley № 25.997 (los vinculados en forma directa e indirecta con el sector). La muestra fue seleccionada al azar y relevó aproximadamente al 30\% de la población, el margen de error, aceptando un nivel de confianza del $95 \%$, se estima en +/- 0,09667.

Una vez realizado el relevamiento se compiló la información obtenida en una base de datos que fue analiza a partir de las técnicas de la estadística descriptiva. Los resultados se evaluaron en función del conocimiento teórico desarrollado por autores referentes con el objetivo de realizar una caracterización del conjunto de emprendedores y emprendimientos locales.

\section{Resultados}

De los 76 emprendedores relevados, el $67 \%$ posee empresas directamente vinculadas con el turismo y el restante $33 \%$ vinculadas en forma indirecta con el sector. El $42 \%$ eligió emprender en alojamientos turísticos, seguido por aquellos que se dedicaron a la gastronomía con el 22\% de las observaciones. La composición de la muestra se describe en la figura 1. 
Figura 1. Distribución de los emprendimientos de acuerdo con la actividad que desarrolla

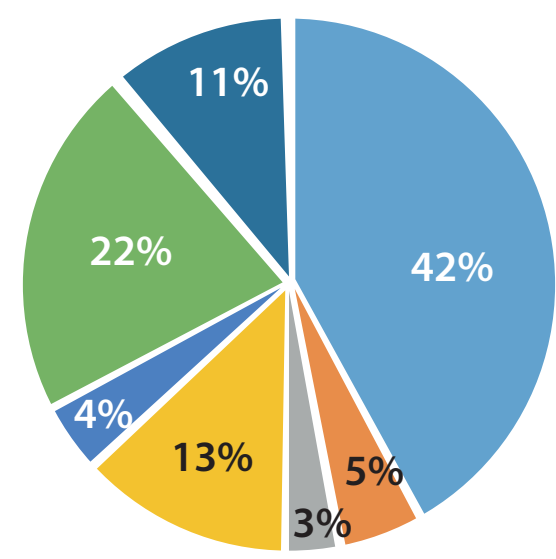

$\begin{array}{ll}\text { - Alojamiento } & \text { "Agencia de viajes y turismo } \\ \text { - Transporte } & \text { "Guía de pesca } \\ \text { - Guía de Turismo } & \text { - Gastronomía } \\ \text { - Otros servicios indirectamente vinculados }\end{array}$

Fuente: Elaboración propia.

\section{Caracterización del emprendedor}

El $66 \%$ de los hombres poseen emprendimientos. No se encontró una relación significativa entre el género del emprendedor y el tipo de emprendimiento, aunque las actividades guía de pesca, agencias de viaje y turismo y transporte son lideradas por hombres. La edad promedio de los dueños es 49,5 años, con un desvío estándar de 13,4 años. El más joven tiene 25 y el mayor tiene 84 años. El 50\% de los casos centrales se trata de adultos entre 39 y 59 años.

En la figura 2 se observa que la mayoría nació fuera de la Provincia de Chubut (66\%), mientras que el $25 \%$ lo hicieron en la localidad de Esquel y el $9 \%$ restante son oriundos de otras ciudades de la provincia. Entre quienes nacieron fuera de Chubut, se encontró que dos de ellos son de nacionalidad chilena, de regiones cercanas a la analizada, y el resto son argentinos, donde el $64,0 \%$ son nacidos en zonas densamente pobladas, como la provincia de Buenos Aires y un $4 \%$ en la Ciudad Autónoma de Buenos Aires. El resto provienen de Patagonia (10\%) y otras provincias del país. Entre quienes nacieron en la provincia y fuera de Esquel, la mayoría son de la región cordillerana. Esta situación refleja la composición social de la localidad, caracterizada por los sucesivos procesos migratorios. 
Figura 2. Lugar de nacimiento del emprendedor

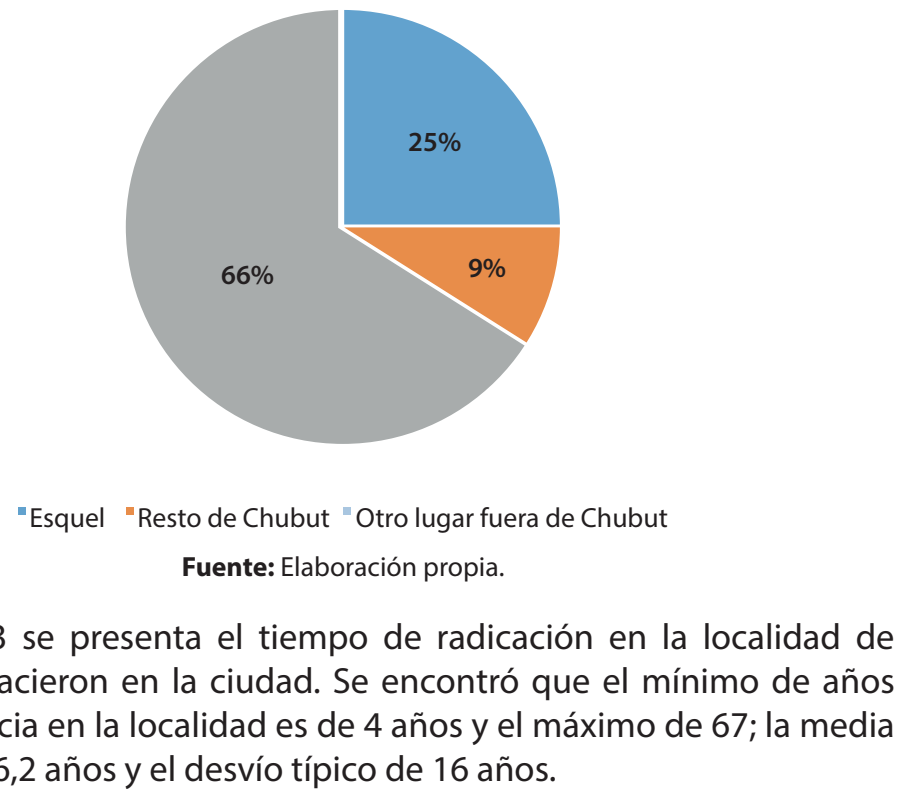

Figura 3. Años de radicación de los emprendedores en Esquel

En la figura 3 se presenta el tiempo de radicación en la localidad de quienes no nacieron en la ciudad. Se encontró que el mínimo de años de permanencia en la localidad es de 4 años y el máximo de 67; la media muestral es 26,2 años y el desvío típico de 16 años.

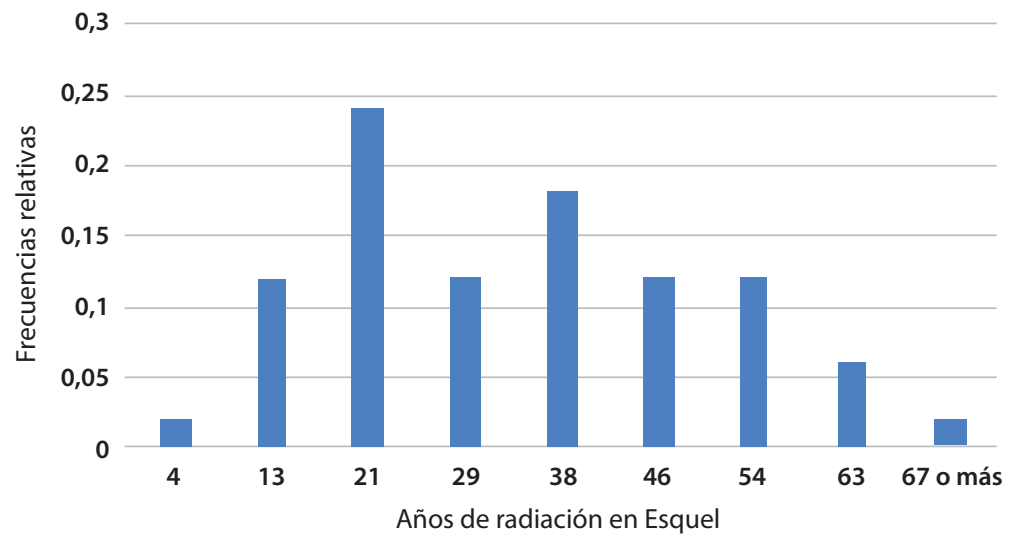

Fuente: Elaboración propia.

El nivel de estudios máximo alcanzado por los emprendedores es elevado en comparación con la media local (Fernández, Ciámpoli y Felipe, 2015). El $34 \%$ posee estudios superiores finalizados y el $21 \%$ inició estudios superiores, aunque no logró finalizarlos. El 25\% tiene el secundario completo y apenas el $20 \%$ tiene un nivel de instrucción inferior al secundario completo (figura 4). Entre quienes tienen título 
universitario y/o terciario completo, encontramos que el 43,5\% de los emprendedores realizaron carreras afines al turismo, como por ejemplo las carreras universitarias de Licenciatura en Turismo o Licenciatura en Administración de Empresas Turísticas. Entre quienes tienen formación técnica y/o terciaria, se encontraron Guías de Turismo, Técnicos en Turismo, Gastronomía e Instructores de Esquí. El 39,1\% realizaron carreras terciarias no vinculadas a la actividad y el $17,4 \%$ realizaron estudios universitarios no relacionados con el turismo.

Figura 4. Nivel de instrucción alcanzado por el emprendedor

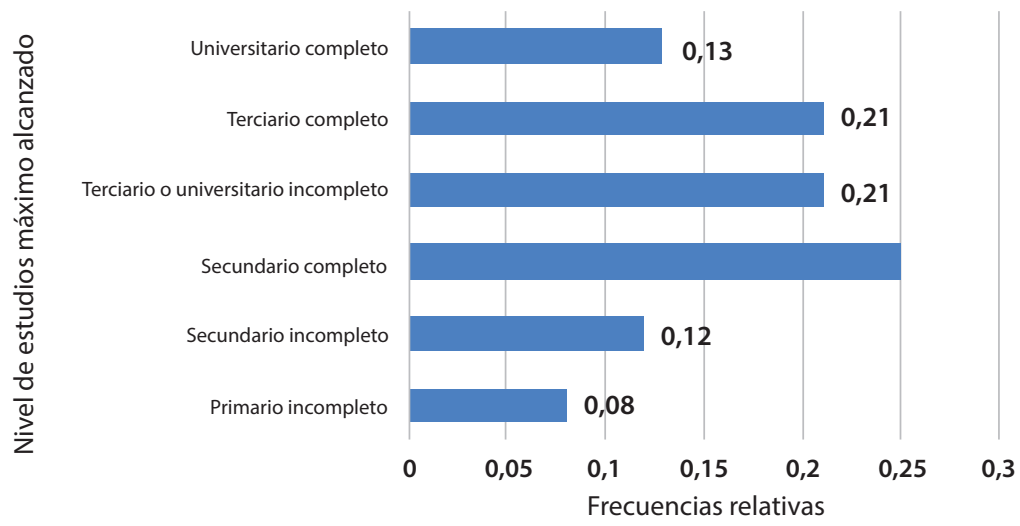

Fuente: Elaboración propia.

En complemento con su nivel de instrucción, el $64 \%$ de los que señalaron que han realizado cursos y/o capacitaciones relacionadas con su emprendimiento lo hicieron fuera del sistema formal. Quienes las realizaron, usualmente, hicieron más de una. La mayor parte de los cursos que realizan se enfocan a mejorar la calidad del servicio, la atención al público, la gestión del negocio, el diseño y/o calidad de productos y la seguridad en las actividades que realizan al aire libre (tabla 1).

Tabla 1. Tipo de curso/especialización que realizan los emprendedores

\begin{tabular}{|l|c|c|}
\hline Tipo de curso/especialización que realizan & Casos & $\begin{array}{c}\text { Frecuencias } \\
\text { relativas }\end{array}$ \\
\hline Enfocadas a rescate y primeros auxilios & 11 & 0,196 \\
Enfocadas a mejorar la calidad del servicio & 8 & 0,143 \\
Enfocadas a tareas administrativas/gestión & 7 & 0,125 \\
Comercialización/Marketing/fijación de precios & 7 & 0,125 \\
Enfocadas a la atención al cliente/público & 5 & 0,089 \\
Cursos de cocina, gastronomía, cerveza, etc. & 5 & 0,089 \\
\hline
\end{tabular}




\begin{tabular}{|l|c|c|}
\hline \multicolumn{1}{|l|}{ Tipo de curso/especialización que realizan } & Casos & $\begin{array}{c}\text { Frecuencias } \\
\text { relativas }\end{array}$ \\
\hline $\begin{array}{l}\text { Enfocadas a la calidad en turismo/ } \\
\text { Buenas prácticas } \\
\text { Guía de pesca y/o turismo }\end{array}$ & 4 & 0,071 \\
$\begin{array}{l}\text { Interpretación del entorno (geología, historia, } \\
\text { intérprete ambiental) }\end{array}$ & 2 & 0,072 \\
\hline $\begin{array}{l}\text { Relacionados con emprendedorismo } \\
\text { Rafting }\end{array}$ & 2 & 0,036 \\
\hline Totales & $\mathbf{5 6}$ & 0,036 \\
\hline
\end{tabular}

Fuente: Elaboración propia.

Quienes no se capacitaron (36\% de los entrevistados), mencionan que los principales motivos por los cuales no lo hicieron fueron: la falta de oportunidad o posibilidad, porque consideran que ya están preparados para lo que hacen y no son necesarias y/o por falta de tiempo.

En promedio, los emprendedores se encontraron trabajando en el sector durante los últimos 14,1 años, con una desviación estándar muestral de 10,9 años. El 50\% con mayor experiencia trabajan en el sector desde hace más de 11 años y el 70\% de los empresarios poseen menos de 14,7 años de experiencia en la actividad (figura 5).

Figura 5. Tiempo que hace que el emprendedor trabaja en el sector turístico

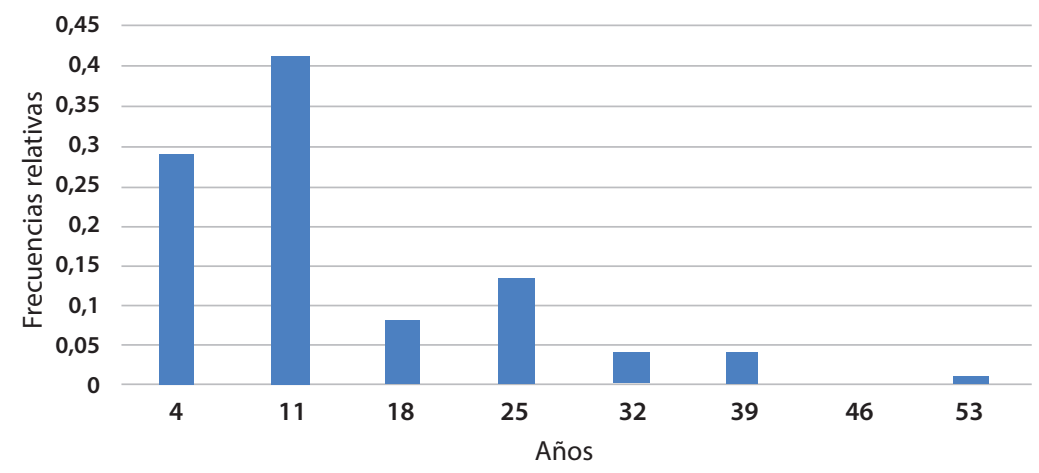

Fuente: Elaboración propia.

La mayoría de los emprendedores tiene experiencia laboral anterior a su emprendimiento turístico, ya sea en trabajos de dependencia y/o en otros emprendimientos propios anteriores. Generalmente, es mayor la experiencia previa de dependencia laboral, si se la compara con la experiencia por cuenta propia. Más del $80 \%$ trabajó en relación de 
dependencia y cerca de la mitad lo hizo en emprendimientos creados con anterioridad al negocio por el que fueron entrevistados (tabla 2). Apenas el $7 \%$ de quienes trabajan en turismo no poseen ningún tipo de experiencia previa, mientras que el $40 \%$ tienen experiencia tanto en relación de dependencia como en emprendimientos anteriores. Al consultar los años de experiencia, se encontró que, en promedio, quienes tienen experiencia laboral en relación de dependencia cuentan con 15,9 años de experiencia, con un desvío muestral de 11,9 años. Estos valores son similares para el caso con experiencia con emprendimientos propios, dónde la cantidad de años promedio de experiencia es 14 y el desvío muestral 11,5 años.

Tabla 2. Experiencia laboral de los emprendedores

\begin{tabular}{|c|c|c|c|}
$\begin{array}{c}\text { Experiencia laboral } \\
\text { (en FR respecto del total) }\end{array}$ & \multicolumn{2}{|c|}{ En relación de dependencia } & \multirow{2}{*}{ Total } \\
\cline { 1 - 2 } En emprendimientos & Si & No & \\
\cline { 1 - 2 } Si & 0,40 & 0,11 & 0,51 \\
No & 0,43 & 0,07 & 0,49 \\
Total & 0,83 & 0,17 & 1,00 \\
\hline
\end{tabular}

Fuente: Elaboración propia.

Del total de los entrevistados, el 71\% señalaron que en la actualidad no se desempeñan en otro trabajo remunerado. El 11\% de los que poseen otra fuente de ingresos trabajaban en el sector privado, el $8 \%$ en el Estado y el $9 \%$ realizaban otras tareas.

Las principales motivaciones que los llevaron a iniciar la actividad son aquellas que tienden a satisfacer necesidades de orden superior: asumir nuevos desafíos, identificar una oportunidad de negocio, buscar un cambio en su estilo de vida, realizar o cumplir un sueño (tabla 3). En este sentido, aunque con menor importancia, mencionan que emprenden para completar sus ingresos o porque valoran la posibilidad de poder tomar decisiones propias.

La motivación de completar los ingresos familiares puede relacionarse con cubrir necesidades de seguridad. Quienes mencionan otros motivos, es porque consideran su emprendimiento como una inversión, son jubilados, quieren mantenerse activos o en contacto con la sociedad y les gusta lo que hacen. Como se trataba de una pregunta de respuestas múltiples, se encontró que, en promedio, eligieron más de 2 motivos entre el total de los planteados. El $80 \%$ de estas respuestas revelan necesidades de orden superior. 
Tabla 3. Motivos por los que decidió emprender

\begin{tabular}{|l|c|}
\hline \multicolumn{1}{|c|}{ Motivos por los que emprende } & Porcentaje \\
\hline Para asumir nuevos desafíos & 0,43 \\
\hline Identifiqué una oportunidad de negocio & 0,39 \\
Porque buscaba un cambio en mi estilo de vida & 0,33 \\
Para realizar cumplir un sueño & 0,32 \\
Para complementar los ingresos familiares & 0,29 \\
Para poder tomar decisiones propias & 0,25 \\
Porque tuve modelos de familiares emprendedores & 0,24 \\
Para continuar con el negocio familiar & 0,13 \\
Porque no logro conseguir un trabajo asalariado & 0,05 \\
Porque fui despedido de otro trabajo & 0,05 \\
\hline Otros & 0,14 \\
\hline
\end{tabular}

Fuente: Elaboración propia.

Para indagar la causa por la que eligieron emprender en el sector turístico, se solicitó a los entrevistados que eligieran hasta tres motivos. La elección se debe, principalmente, a su formación y/o especialización y a la experiencia en el sector (gráfica 6). Esto podría vincularse con motivaciones de autorrealización y estima, debido a que el individuo elige realizar actividades en un contexto donde se sienten preparados para desplegar sus competencias personales (saber y poder hacer).

En segundo plano, aparecen como factores importantes la herencia del negocio, la tradición familiar en la actividad y otros motivos, que son su preferencia personal (porque les gusta), o de tipo altruistas como procurar el cuidado de los atractivos y recursos naturales de la zona y porque consideran que invirtiendo en el sector ayudarían al desarrollo económico y de la actividad en la localidad. Las categorías señaladas se encuentran en el orden de necesidades superiores, de carácter social, tales como el sentido de pertenencia, afiliación y participación en la sociedad.

Los datos observados sobre las variables de nivel de instrucción, interés en capacitarse o prepararse para mejorar la calidad del servicio brindado, la experiencia laboral previa, ya sea en el sector público y/o privado, y las causas por las que decidieron emprender, podrían indicar que se trata de emprendedores que cuentan con elevado capital social enfocado al desarrollo del negocio. 
Figura 6. Motivo por el que eligió una actividad vinculada al turismo

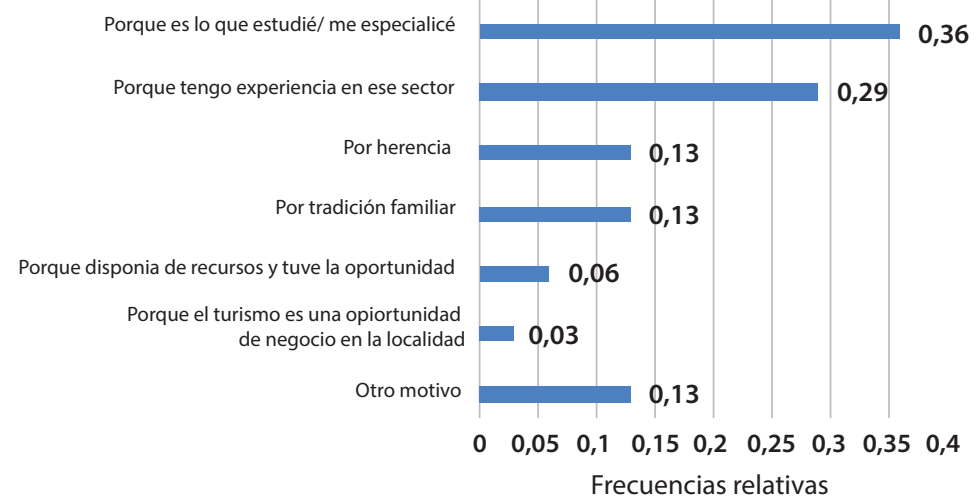

Fuente: Elaboración propia.

Los aspectos que el emprendedor valoró en relación con su actividad independiente es que le permite progresar o crecer económicamente, le brinda independencia y autonomía, la posibilidad de obtener el fruto de su propio trabajo, la flexibilidad horaria y la posibilidad de poner en práctica sus propias ideas. También mencionan, aunque con menor importancia, que pueden estar al frente de una organización y/o dirigir un grupo de personas, y tienen la posibilidad de invertir su patrimonio (figura 7). En promedio, los entrevistados eligieron 3,1 motivos por los que valoran tener su propio negocio. Aquellos que escogieron otro motivo lo relacionan con su gusto por estar en contacto con la gente.

Figura 7. Lo que valora de tener su emprendimiento

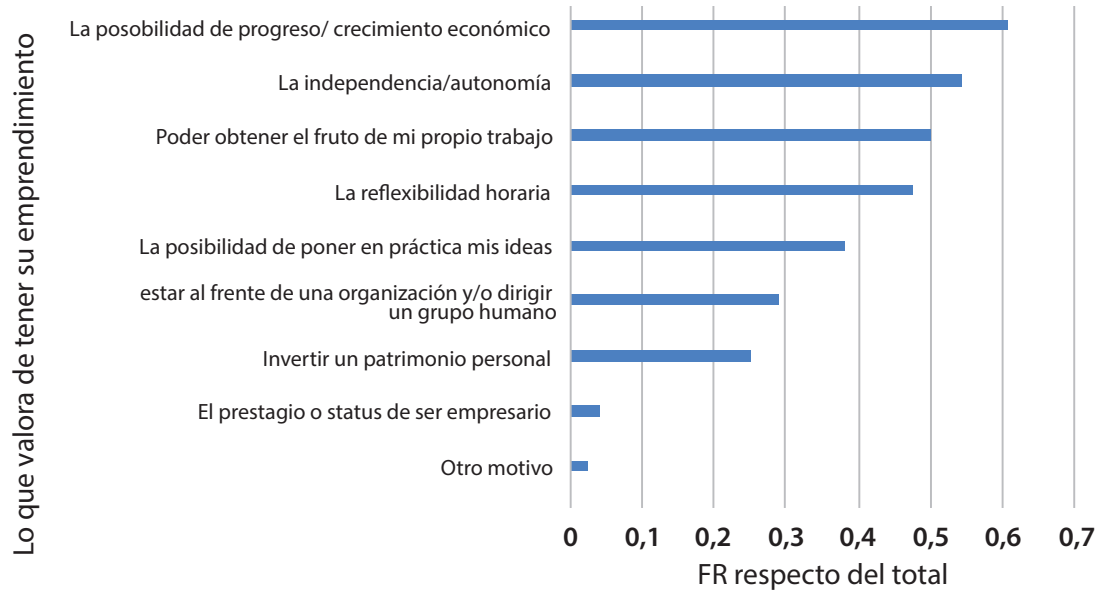

Fuente: Elaboración propia. 
Finalmente, se quiso conocer si se consideraban emprendedores, de acuerdo con la definición propuesta en la metodología (Cesar et al. 2016: 4). El $82 \%$ se reconoció emprendedor, mientras que el $13 \%$ no se considera como tal. El 5\% no sabe o no responde. Quienes se consideraron emprendedores mencionan o rescatan lo siguiente:

El $14,5 \%$ de los entrevistados que realizan comentarios acerca de su elección coinciden con la definición o se sienten identificados plenamente con ella, mientras que el $44,8 \%$ se identificó con algunas de las ideas que se explicitan en ella. En general, quienes proponen aportes, rescatan factores relacionados con su personalidad, sus sentimientos o actitudes, señalando que la definición puede ampliarse en esta dimensión. Las características que rescatan se relacionan con la perseverancia, creatividad, disposición a asumir riesgos, la capacidad de aceptar desafíos, la autosuficiencia y autosuperación.

\section{Caracterización de los emprendimientos}

Se encontró que, en promedio, las empresas tienen 16 años, observándose un desvío muestral de 11,8 años. La figura 8 muestra la edad de las empresas considerando su nacimiento el año de su fundación. La mayor parte de las mismas (el 75\% o más) tienen menos de 21 años, siendo la mediana 11 años. Es menos frecuente la creación de empresas con mayor edad, donde el emprendimiento más antiguo fue fundado hace 57 años.

Figura 8. Diagrama de caja según la edad de la empresa

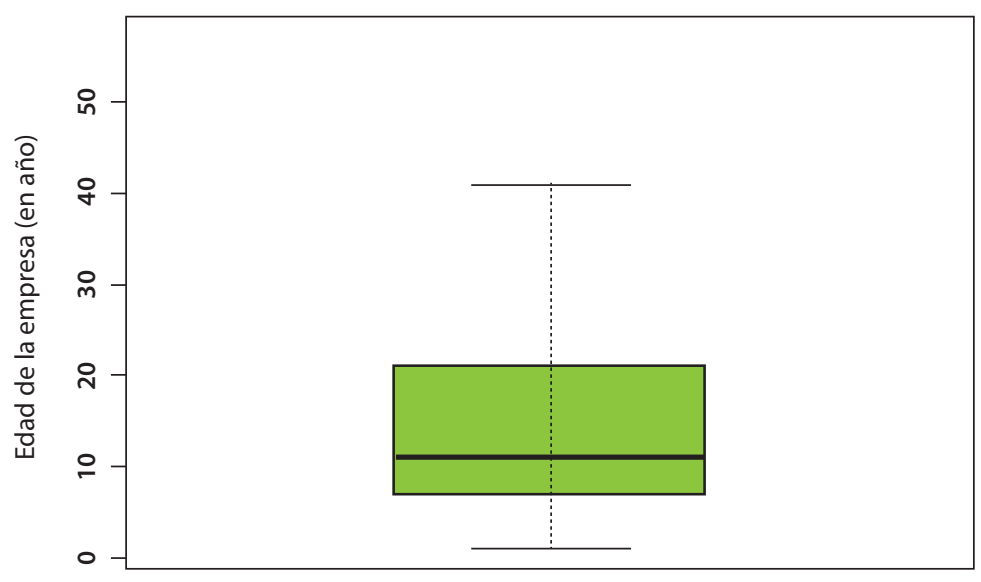

Fuente: Elaboración propia.

El tipo societario más frecuente pertenece al grupo de las empresas unipersonales, que representa el $66 \%$, seguido de las sociedades de responsabilidad limitada con un $21 \%$. El resto se trata de otro tipo de 
organizaciones con fines de lucro (Sociedades de Hecho, Colectiva y Sociedad Anónima).

Se observaron varios emprendimientos en los que trabaja solamente el dueño (el 26\% de los casos). El promedio de personas que trabajaron en los emprendimientos turísticos de la ciudad acompañando a los emprendedores es 3,4 personas, y el desvío estándar muestral es de 5,6 personas. El 49\% de los emprendimientos que menos personal contrató, tiene hasta 4 personas trabajando, y el $24 \%$ de las empresas contrató hasta 2 empleados. La empresa que tuvo mayor cantidad de personal, cuenta con 39 empleados (figura 9).

Figura 9. Personas trabajando actualmente en la empresa, además del emprendedor

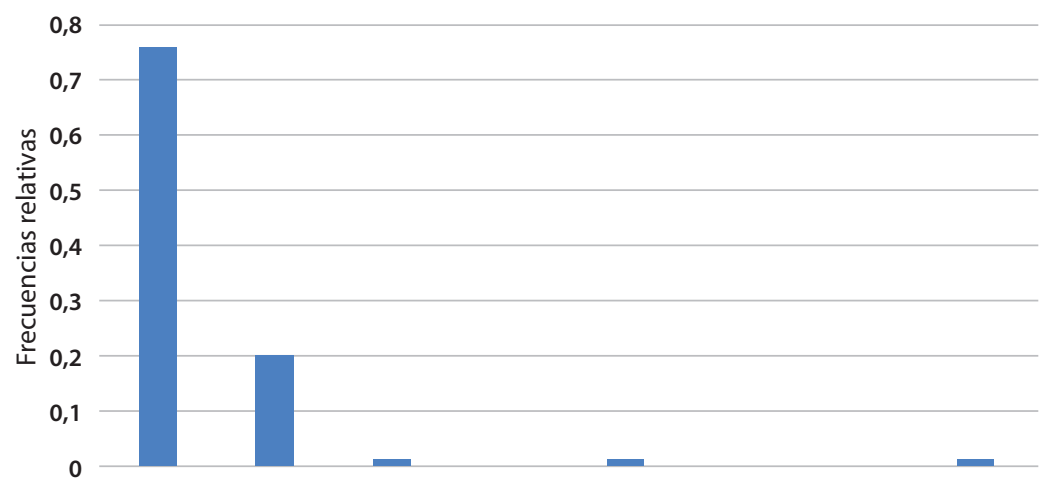

Cantidad de personal, además del emprendedor

Fuente: Elaboración propia.

El $62 \%$ de las empresas que emplearon personal tuvieron a algún familiar trabajando en la empresa. En promedio, empleó a 1,7 familiares, donde el desvío estándar muestral fue 0,96. La empresa que más parientes empleó tiene a 5 de ellos trabajando en el emprendimiento y la que menos contrató a 1. El parentesco de los familiares que trabajaban en la empresa fue la pareja o cónyuge (61\%), los padres (33\%) o los hijos (30\%). Con menor importancia mencionaron a los hermanos (18\%) y otros parientes (9\%), siendo estos últimos cuñados o sobrinos.

De los 76 encuestados que formaron parte de la muestra, el 47,4\% poseen solo un emprendimiento, el 19,76\% y el $9 \%$ indicaron que tienen otro negocio y dos o más emprendimientos respectivamente en el mismo sector. El 14,5\% expresó que tienen una empresa perteneciente a otro sector de la economía y el $3 \%$ que posee dos negocios más en otro sector.

La mayor parte de las empresas son micro y pequeñas empresas familiares, con algunos pocos casos en los que se encontraron emprendimientos 
con una mayor cantidad de empleados. Algunos emprendedores tienen más de un emprendimiento en forma simultánea, complementando las actividades que realizan dentro y fuera del sector turístico.

\section{Conclusiones y discusión}

En relación con la caracterización de los emprendedores, varios son propietarios de más de un emprendimiento, extendiéndose en forma horizontal en el sector, y en otros sectores de la economía local. Se trata, en mayor medida, de hombres nacidos fuera de la provincia de Chubut, que tienen en promedio 49,5 años de edad y 26,2 años de radicación en la localidad. Poseen un nivel de estudios elevado y muestran interés en capacitarse en el área que eligieron. La mayoría tiene experiencia laboral previa y en la actualidad solventan a su familia, exclusivamente, con los ingresos que obtienen de sus negocios. El $82 \%$ se identifica como emprendedor. Eligen el turismo porque es lo que estudiaron, por experiencia laboral previa o porque heredaron el negocio. Valoran los beneficios del trabajo por cuenta propia: posibilidad de progreso, independencia, obtener el fruto de su trabajo, flexibilidad horaria, etc.

Se asocia el emprender con el desafío, la identificación de oportunidades de negocios, la búsqueda de un cambio en su estilo de vida y la posibilidad de cumplir un sueño. El $80 \%$ de las motivaciones seleccionadas por los emprendedores se orienten a cubrir necesidades de orden superior, cuyas motivaciones más sobresalientes se relacionan con la superación personal y la realización, antes que por causas negativas, como no conseguir empleo o haber quedado desempleado. De las características que mencionan, podría señalarse que la mayoría se trata de emprendedores por oportunidad, que desarrollaron emprendimientos de tipo estilo de vida o style life siguiendo la clasificación que realiza Lebendiker (2013). Desean mantener un determinado estilo de vida y su principal objetivo no gira en torno a la obtención de un beneficio extraordinario, sino, más bien, a la realización de una actividad que los gratifique en lo personal y que cubra sus expectativas en términos del tiempo que le dedican y los conocimientos y experiencia que poseen. Se observan características que indican que poseen un elevado capital social que pueden transferir a sus empresas.

Con respecto a las empresas, la mayor parte son medianas y pequeñas empresas de tipo familiar, puesto que son pocos los emprendimientos con mayor cantidad de empleados. La creación de empresas en el sector es un fenómeno que se presentó con mayor fuerza entre 1996 - 2010, período en el que se crearon, aproximadamente, el 50\% de las empresas turísticas que fueron analizadas. Los emprendimientos con mayor participación en el sector son los pertenecientes al rubro alojamiento (42\%) y gastronomía (22\%). 
Las características observadas son acordes con la descripción que realiza Parra y Calero (2006: 545). Estas características también son rescatadas por Del Castillo, López y Ramos (2013: 155), al afirmar que quienes emprenden desean mejorar su bienestar y aplicar los conocimientos técnicos adquiridos, más que la búsqueda del éxito económico, siendo la aspiración de la mayor parte de los emprendedores la de crear una empresa que crezca sin apuros ni sobresaltos.

Aparece como un hecho a destacar que entre los entrevistados no se reconozca al turismo como una oportunidad de negocio, aunque mencionan que lo que los motivó a emprender fue que identificaron una oportunidad de negocio. Este hecho plantea al equipo de investigadores un desafío para identificar los factores que hacen que se presenten resultados divergentes en este tema. ¿La diferencia se encuentra entre la etapa inicial donde ellos observaron al turismo como una oportunidad de negocioy deciden emprender, y la etapa de desarrollo del emprendimiento en la que existen factores políticos, económicos, culturales y sociales del contexto que afectan esa mirada inicial? ¿Podrían ser las políticas estatales o el vínculo dentro del sector turístico y/o de este con la sociedad lo que ocasiona esta discrepancia?

Otra explicación puede brindarla la existencia de debilidades gerenciales que posee este tipo de empresas, la escasa experiencia comercial y las motivaciones basadas en el estilo de vida que se asocian a beneficios subóptimos, como también el acceso limitado a financiamiento y al entorno regulatorio institucional que muchas veces inhibe el crecimiento y la entrada de nuevas firmas al sector o que causan problemas para la supervivencia (Ateljevic, 2009: 149).

La alta potencialidad de capital social individual entre los emprendedores no implica que esta habilidad sea replicable a nivel colectivo. Esto se podría indagar en términos del entorno o entramado social de la comunidad a través de la capacidad de conformación de redes, la cooperación entre empresas, la confianza en el otro y el acompañamiento institucional. La definición de capital social planteada desde una visión expansionista, lo precisa como los aspectos de la organización social, tales como la confianza, normas y redes, que pueden mejorar la eficiencia de una sociedad al facilitar la acción coordinada (Ostrom y Ahn, 2003: 166). Este análisis escapa a los objetivos del presente trabajo, pero podría abordarse en futuras investigaciones.

\section{Agradecimientos}

Se agradece el aporte realizado por los estudiantes de la Cátedra de Estadística cohorte 2017, de la Facultad de Ciencias Económicas de 
la Universidad Nacional de la Patagonia San Juan Bosco, Sede Esquel, en el relevamiento, carga de la base de datos y procesamientos de las preguntas incluidas en el cuestionario.

\section{Referencias bibliográficas}

1. ARGENTINA. Senado y Cámara de Diputados de la Nación Argentina. (2014). Ley Nacional de Turismo 25.997. Buenos Aires.

2. ARGENTINA. Cámara de Comercio, Producción, Industria y Turismo del Oeste del Chubut (CCPITOCH). (2006). Esquel 100 años. Una Empresa, un siglo. Esquel, Chubut: Cámara de Comercio, Producción, Industria y Turismo, Provincia del Chubut. $151 \mathrm{pp}$

3. ARGENTINA. Subsecretaría de Cultura y Educación. (2009). Esquel 100 años. Crónicas del primer centenario en el bicentenario de la nación argentina. Esquel, Chubut: Comisión Pro Museo Histórico Esquel. Subsecretaría de Cultura y Educación, Municipalidad de Esquel. 388 pp.

4. ATELJEVIC, Irena y DOORNE, Stephen. (2000). 'Staying Within the Fence': Lifestyle Entrepreneurship. Tourism, Journal of Sustainable Tourism, Vol. 8, №. 5. Pp. 378392.

5. ATELJEVIC, Jovo. (2009). Tourism Entrepreneurship and Regional Development (pp. 141-179). En: ALTEJEVIC, Jovo et al. (Eds.) Tourism and Entrepreneurship. International Perspectives. Oxford (UK): Elsevier.

6. ATELJEVIC, Jovo \& LI, Lan. (2009). Tourism Entrepreneurship - Concepts and Issues. (pp. 10-32). En: ALTEJEVIC, Jovo et al. (Eds.). Tourism and Entrepreneurship. International Perspectives. Oxford (UK): Elsevier.

7. CÉSAR, Rubén, LÓPEZ, Silvia Mabel, RAMÍREZ, María Celeste, FERNÁNDEZ, María Victoria, GAUNA, Mariana, GAJARDO, Rodrigo, DOMÍNGUEZ, Carlos, FELIPE, Jenifer y GARRIDO, Tamara. (2016). Aportes al análisis del proceso emprendedor en el Sector Turístico de Esquel. Ponencia en VII Jornadas Patagónicas de Investigación y II Jornadas Patagónicas de Extensión en Ciencias Económicas. Trabajos de Investigación y Extensión. Memoria digital. Esquel, Chubut: Universidad Nacional de la Patagonia San Juan Bosco. Disponible en Internet: https://bit.ly/2KRicMd

8. CHIAVENATTO, Idalberto. (2001). Administración de Recursos Humanos. 5a ed. Bogotá: Mc Graw Hill. 699 pp.

9. DEL CASTILLO, Martín, LÓPEZ, Andrés y RAMOS, Daniela. (2013). El fenómeno emprendedor en Argentina: Características, Motivaciones y obstáculos (pp. 123162). En: KANTIS, H. (Coord.) ¿Emprendimentos dinámicos en américa del sur? La clave es el (eco) sistema. Montevideo (Ur.): Red Mercosur de Investigaciones Económicas. 
10. FERNÁNDEZ, María Victoria, CIÁMPOLI, María Cecilia y FELIPE, Jenifer. (2015). Aplicación de la metodología propuesta por la Asociación Argentina de Marketing para la medición del Nivel Socioeconómico de la localidad de Esquel. Ponencia en VI Jornadas Patagónicas de Investigación y I Jornadas Patagónicas de Extensión en Ciencias Económicas. Trabajos de Investigación y Gestión. Memoria digital. Esquel, Chubut: Universidad Nacional de la Patagonia San Juan Bosco. Disponible en Internet: https://bit.ly/2xm3rls

11. GÁMEZ, Alba Eritrea, ÁNGELES, Manuel y JUÁREZ, Eduardo. (2013). Turismo y emprendedurismo en regiones rurales de México: una crítica al rol del capital social en el desarrollo local. El caso del oasis de los Comondú. Revista de Investigación en Turismo y Desarrollo Local, Vol. 6, № 14. Pp. 1-26. Disponible en Internet: https://bit.ly/2No5g2h Consultado: 05.12.2018.

12. GARTNER, William. (1988). "Who is an entrepreneur?" Is the wrong question. American Journal of Small Business, Vol. 12, № 4. Pp. 11-32. https://doi. org/10.1177/104225878801200401

13. KANTIS, Hugo y FEDERICO, Juan. (2007). Crisis y renacimiento emprendedor en la Argentina: evidencias y algunos interrogantes. Boletín informativo Techint, № 322. Pp.13-28. Disponible en Internet: https://bit.ly/2FLSJQa Consultado: 07.08.2018.

14. LEBENDIKER, Adrián Leonardo. (2013). Emprender, ¿sí o no? Cómo conocer el propio potencial emprendedor (pp. 11-28). En: SVARZMAN, Gustavo (Coord.). Claves para emprendedores: conceptos básicos para planificar y desarrollar tu proyecto. Buenos Aires (Arg.): Ministerio de Desarrollo Económico del Gobierno de la Ciudad de Buenos Aires.

15. OSTROM, Elinor y AHN, T. K. (2003). Una perspectiva del capital social desde las ciencias sociales: capital social y acción colectiva. Revista Mejicana de Sociología, Vol. 65, № 1. Pp. 155-233.

16. PARRA, Eduardo y CALERO, Francisco. (2006). Gestión y Dirección de Empresas Turísticas. $2^{\circ}$ ed. Madrid: Mc Graw Hill. 545 pp.

17. ROBBINS, Stephen y JUDGE, Timothy. (2009). Comportamiento Organizacional. $1^{\circ}$ ed. Ciudad de Méjico: Pearson Educación. 752 pp.

18. ROMÁN, Ruth Esperanza y SMIDA, Alí. (2009). El rol del capital social del emprendedor en la expansión exitosa de una start-up. El caso de Hipertexto Ltda. - “La librería de la U”. Estudios Gerenciales, Vol. 25, № 113. Pp. 15-33.

19. SHANE, Scott, LOCKE, Edwin \& COLLINS, Christopher. (2003). Entrepreneurial Motivation. Human Resource Management, Vol.13, № 2. Pp. 257-279. Disponible en Internet: https://bit.ly/323SR7e Consultado: 14.08.2018.

20. SHAPERO, Albert. (1984). The Entrepreneurial Event (pp. 21-40). En: KENT, Calvin. (Ed.). The environment of entrepreneurship. Lexington: Lexington Books. 
21. SOLVOLL, Solvi, ALSOS, Gry Agnete \& BULANOVA, Oxana. (2015). Tourism Entrepreneurship - Review and Future Directions. Scandinavian Journal of Hospitality and Tourism, Vol. 15, № 1. Pp. 120-137.

22. TORRADO, Susana. (1992). Estructura Social de la Argentina: 1945-1983. Buenos Aires: Ediciones de la Flor. 556 pp.

23. VECIANA, José María. (1999). Creación de empresas como programa de investigación científica. Revista Europea de dirección y economía de empresas, Vol. 8, № 3. Pp.11-36.

Para citar este artículo:
FERNÁNDEZ, María Victoria, RAMÍREZ, María Celeste, LÓPEZ, Silvia Mabel, GAUNA, Mariana y CESAR, Rubén Ángel. (2019). Caracterización de emprendedores y emprendimientos del sector turístico de Esquel, Argentina. Teuken Bidikay, Vol. 10, № 15. Pp. 159-181. doi:10.33571/teuken.v10n15a7

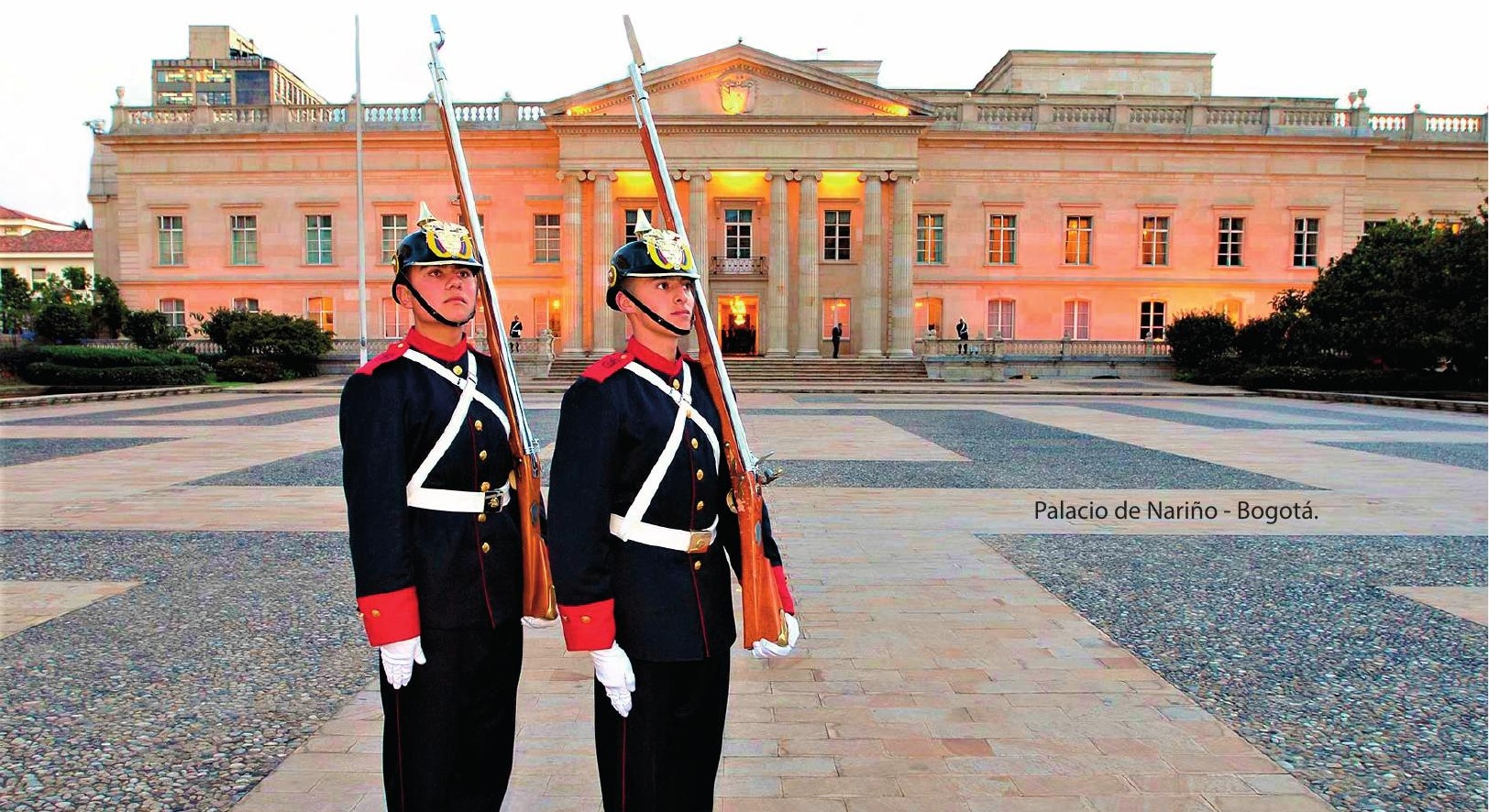


En estas coloridas calles, ahora atestadas de turistas europeos y norteamericanos, están representados los ancestros indígenas, el dolor de la invasión, el letargo de la colonización, los ideales de la Ilustración encarnados en Antonio Nariño y Simón Bolívar, el grito de Independencia, las tramas de la República, la violencia de los años cincuenta, y la de hoy, que va mutando en guerrillas, paramilitares... El barrio La Candelaria es el lugar más histórico y representativo de Bogotá; hablar de él es adentrarse en un universo complejo de pluralidades humanas, de reyertas independentistas y nostálgica arquitectura, que a veces es colonial, con tribunas y balcones elaborados en madera, y otras veces es republicana, que se distingue, principalmente, por las edificaciones que albergan los organismos estatales. Sus calles (re)descubren el devenir humano e histórico de la capital colombiana, y representan la resistencia ante la modernización y el deseo de que algún día los ciudadanos libres dignificarán las instituciones y el espíritu de la República.

Martha Elena Martínez V.

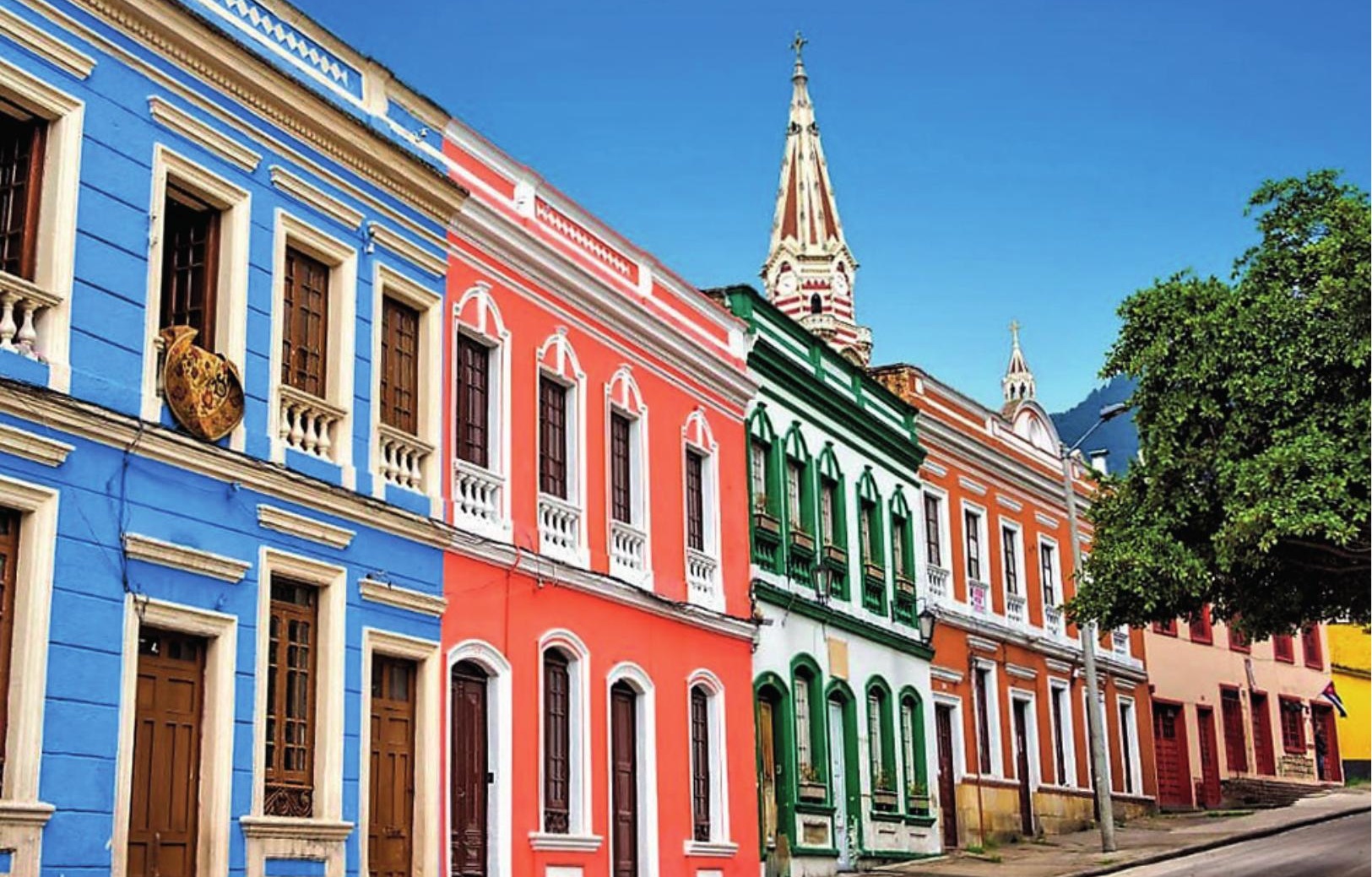

